Au début de I'année, la FMH a lancé le projet pilote du monitorage de la qualité. Il est temps de le présenter aux lecteurs du Bulletin des médecins suisses! Le présent article montre quel est l'objectif poursuivi par le domaine Données, démographie et qualité (DDQ) au moyen de ce projet pilote, qui sont les acteurs du projet, ce qui a été élaboré jusqu'à présent et quels défis caractériseront les prochaines étapes du projet.

Département DDQ de la FMH

\title{
Monitorage de la qualité - Le corps médical assure la qualité
}

\author{
Lea Schläpfer, Daniel Herren, \\ Martina Hersperger
}

\section{Correspondance:} Lea Schläpfer FMH, Département DDQ Elfenstrasse 18 CH-3000 Berne 15 Tél. 0313591111 Fax 0313591112 ddq@fmh.ch

\author{
Le projet pilote du monitorage de la qualité \\ en bref \\ Le monitorage de la qualité a pour but \\ - de répertorier et de présenter les activités en \\ matière de qualité des médecins du secteur \\ ambulatoire \\ - d'évaluer ces activités sur le plan coûts/utilité \\ - de définir des indicateurs qui reproduisent de \\ manière judicieuse la qualité des traitements \\ - d'élaborer un modèle propre à soutenir et à \\ promouvoir l'assurance qualité médicale
}

Mme Rochat souffre depuis longtemps de maux de tête et de vertiges. Elle se met à la recherche d'un traitement médical adéquat. Comme elle vient de déménager, elle choisit la voie pragmatique et se présente au cabinet médical le plus proche. Elle s'attend à ce que le Dr B., médecin généraliste, prenne suffisamment de temps pour examiner son cas. Elle part du principe qu'il posera le bon diagnostic, lui proposera un traitement adéquat et saura l'envoyer chez le spécialiste compétent, le cas échéant. Bref, Mme Rochat compte sur un traitement médical de qualité élevée. Le Dr B. est également très exigeant envers lui-même. De ce fait, il s'engage quotidiennement en faveur de la qualité dans son cabinet. Entre autres choses, il contrôle régulièrement son stock de médicaments et l'état de son laboratoire, demande à ses patients s'ils sont contents, suit des cours de formation continue pour être au top niveau des connaissances médicales et discute des cas difficiles dans le groupe Balint.

\section{Activités médicales pour assurer la qualité}

En Suisse, l'assurance qualité et le développement de la qualité sont traditionnellement ancrés dans l'exercice de la médecine. Aujourd'hui, les activités du corps médical n'ont jamais été aussi nombreuses en la matière. Car, pour les partenaires de la santé, avant tout pour les patients et les médecins eux-mêmes, il va de soi de satisfaire à l'exigence, inscrite dans la LAMal, d'assurer et d'attester la qualité des traitements médicaux. Néanmoins, les revendications de la politique et du public pour une augmentation de la qualité des traitements médicaux montrent que les activités menées par le corps médical doivent être visibles, d'autant plus qu'elles existent déjà. En outre, il est nécessaire d'évaluer ces activités sur le plan des charges qu'elles induisent et de l'utilité qui en résulte, conformément aux connaissances professionnelles du corps médical. Puisque ce sont les médecins qui effectuent la partie principale de ces activités, il est indispensable qu'ils soient associés à la prise de décision pour définir quelles données médicales devront être saisies, évaluées et publiées.

\section{Démarrage du projet pilote}

Avec le projet pilote de monitorage de la qualité, le corps médical assume un rôle clé dans les discussions sur la qualité des soins médicaux fournis dans le domaine ambulatoire. Durant la phase pilote, le projet de monitorage de la qualité s'étendra à la médecine de famille, à la psychiatrie et probablement à l'orthopédie. Dans un premier temps, les sociétés de discipline impliquées enregistrent les multiples activités réalisées par leurs membres en faveur de la qualité. L'objectif est de reproduire le paysage des démarches qualité effectuées par le corps médical dans ces trois domaines. Le principe est simple: les médecins déclarent quelle activité ils ont choisie parmi celles proposées par leur société de discipline en matière de qualité. Comme chaque activité est différente, elle est accompagnée d'une évaluation quant à son rapport utilité/coûts. Par exemple, une certification demande beaucoup de travail et a une autre utilité qu'un sondage sur la satisfaction des patients. L'auto-déclaration permet d'obtenir une image des activités de qualité au sein d'une société de discipline, et chaque médecin peut refléter ses activités au sein de sa discipline. La valeur de cette saisie est claire: mettre en œuvre un outil d'information dans la jungle des démarches qualité. Chaque médecin reçoit des informations individuelles lui indiquant dans quels domaines il a déjà beaucoup œuvré et dans lesquels il doit éventuellement combler des lacunes. 
Données et transparence relatives à la qualité Il s'agit de promouvoir la transparence non seulement dans les activités en faveur de la qualité mais aussi dans la qualité même du traitement médical [1]. Pour être efficace, appropriée et économique, la fourniture de soins a besoin de mesures judicieuses permettant de déterminer la qualité du traitement. De ce fait, une partie du projet pilote vise à déterminer des indicateurs propres à créer la transparence en matière de la qualité parallèlement aux activités de qualité menées dans son cadre. Cette transparence a pour but de donner des informations sur la qualité, utiles à tous les acteurs de la santé - en particulier aux médecins, patients, assureurs et autorités. Les indicateurs sont judicieux uniquement s'ils représentent véritablement la qualité du traitement. Mais quelles sont les données qui permettent de se prononcer sur la qualité d'un traitement médical? Cette tâche, à savoir la définition d'indicateurs appropriés, n'est certainement pas plus facile dans le secteur ambulatoire qu'en milieu hospitalier. Selon les premières expériences faites dans le secteur ambulatoire, il faut que les indicateurs soient impérativement définis avec les spécialistes en la matière pour permettre d'évaluer la qualité des actes médicaux. Ce défi se pose aussi à la Commis- sion chargée du projet pilote Monitorage de la qualité, laquelle est composée de la direction du projet, de délégués des sociétés de discipline concernées et d'experts.

\section{Charges, coûts et incitation pour la qualité}

Garantir la qualité et l'attester coûte de l'argent et prend du temps. Même si cette démarche profite grandement aux patients, qui doit payer les efforts accomplis dans ce domaine? On ne sait toujours pas comment alléger la charge administrative supplémentaire assumée par le corps médical pour procéder aux mesures de la qualité, ni comment la gérer sur le plan administratif et l'indemniser. Un grand point d'interrogation se cache derrière le financement de l'assurance qualité médicale, d'autant plus que l'augmentation constante des coûts de la santé induit la question, importante sur le plan politique, de savoir s'il ne faudrait pas aussi récompenser les médecins qui fournissent de meilleures prestations. L'affirmation selon laquelle les activités en faveur de la qualité, allant de soi, sont contenues dans les tarifs existants s'oppose à l'avis selon lequel il est naturel de mieux indemniser le travail bien fait. En tous les cas, le système de pénalisation prévu dans la LAMal nous semble inap-
1 cf. Transparence de la qualité Document de base du GTQ FMH: Bull Méd Suisses. $2009 ;(90) 26 / 27: 1037-40$.

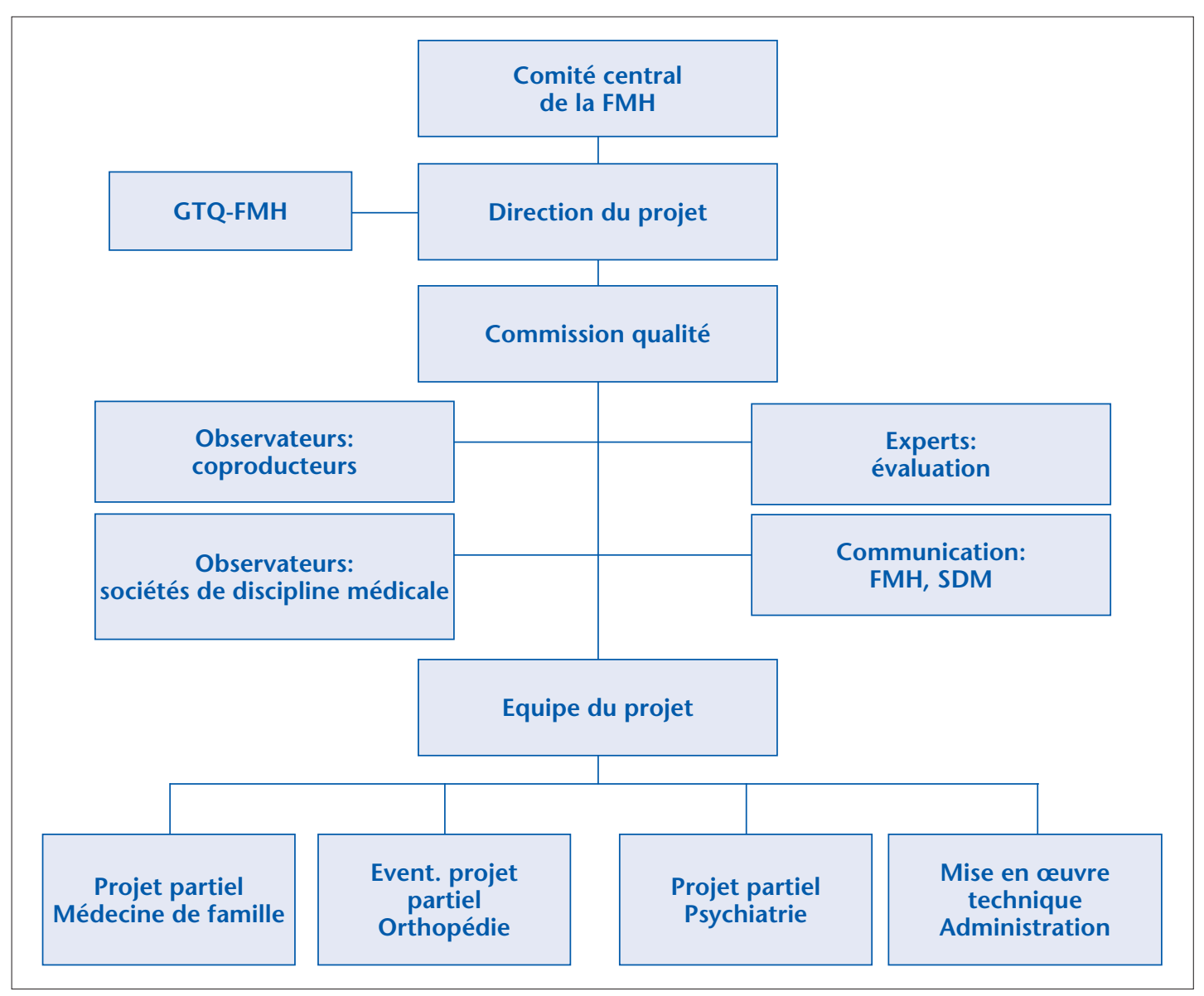

Organisation du projet 
proprié pour motiver les médecins à mener plus d'activités en matière de qualité que la loi le prescrit. Dans le cadre du projet de monitorage de la qualité, le domaine Données, démographie et qualité (DDQ) propose des solutions et évalue les incitatifs et méthodes susceptibles de soutenir les activités du corps médical.

\section{Consultation des sociétés de discipline médicale}

L'assurance qualité est en premier lieu l'affaire des sociétés de discipline médicale. Elles seules sont en mesure de définir les activités qualité de manière judicieuse et utile. Depuis le mois de mars dernier, les groupes de travail ont élaboré des grilles pour la saisie des activités en matière de qualité dans le cadre de leurs spécialisations respectives. Ces grilles sont maintenant mises au point pour devenir des questionnaires en ligne disponibles sur l'internet. Avec l'assistance du service informatique, le département DDQ lancera à fin octobre / début novembre un premier test avec un petit contrôle ponctuel par domaine pilote. Au cours du premier semestre 2010, les membres des sociétés de discipline impliquées seront invités à déclarer leurs activités en faveur de la qualité au moyen du questionnaire déposé sur le portail internet myFMH. Ici aussi, le rôle de ces sociétés est d'importance: ce n'est qu'avec leur aide que la FMH parviendra à motiver ses membres à participer à cette enquête. Une fois terminées, les évaluations serviront non seulement à établir un rapport sur les résultats de l'enquête dans l'intérêt collectif du corps médical, mais permettront aussi à chaque médecin en particulier de profiter de son engagement et d'obtenir un feed-back individuel sur ses activités dans le domaine de la qualité.

\section{Encourager le dialogue}

\section{dans la santé publique}

La FMH s'entend d'une part comme coordinatrice des activités menées par les sociétés de discipline médicale. En tant qu'organisation faîtière, elle défend d'autre part le corps médical face aux organisations partenaires de la santé. De ce fait, la FMH a invité ses organisations à accompagner le projet pilote en tant qu'observateurs. A l'heure actuelle, l'Office fédéral de la santé publique, la Conférence des directeurs canto- naux de la santé, $\mathrm{H}+$, santésuisse, la Fédération suisse des patients, la Fondation pour la sécurité des patients, la Commission des tarifs médicaux/SUVA ainsi que les assurances-maladie Helsana, CSS et Concordia sont représentés dans le groupe d'observation. Les présidents des sociétés de discipline impliquées dans le projet ainsi que les présidents du VEDAG et du Collège pour la médecine de famille sont également membres de ce groupe d'observation. A la mi-juin, la direction du projet a rencontré les observateurs au Secrétariat général de la FMH et les a informés des avancées du projet. Une discussion fructueuse a ensuite porté sur les objectifs de cette entreprise, et notamment sur l'élaboration d'un modèle d'incitatifs judicieux pour encourager la qualité des soins médicaux. Les participants ont salué cette rencontre entre partenaires de la santé et souligné leur intérêt pour des tels échanges. Le projet de monitorage de la qualité est ainsi une plateforme favorable à un dialogue productif sur la qualité des soins médicaux.

\section{Direction du projet de monitorage de la qualité}

Ce projet est dirigé par le département Données, démographie et qualité (DDQ) de la FMH, notamment par le Dr Daniel Herren, membre du Comité central et responsable du domaine, ainsi que par Martina Hersperger, responsable de département. Le département DDQ est chargé de saisir et d'analyser les données et d'en communiquer les résultats au sein de la FMH et vers l'extérieur.

Vous trouverez de plus amples informations sur le projet pilote de monitorage de la qualité sous: www.fmh.ch/themen/qualitaet/projekte.html. Contact: ddq@fmh.ch

A propos, le Dr B. estime que les activités quotidiennes pour assurer la qualité dans son cabinet médical vont de soi. En les déclarant sur le portail des membres myFMH, il participe à la démarche visant à présenter au public le travail effectué par l'ensemble du corps médical en faveur de la qualité. Des données représentatives sont des données pertinentes aptes à former une base d'arguments pour une discussion judicieuse sur la qualité. 